\title{
Evaluación de la carga de fallo por tracción diagonal en vigas de hormigón armado sin cercos a través del estudio del efecto de tamaño
}

\author{
Assessment of diagonal-tension failure load in reinforced concrete beams \\ without stirrups through the study of size effect
}

J. R. Carmona ${ }^{(*)}$, G. Ruiz ${ }^{(*)}$

\section{RESUMEN}

El presente trabajo propone una expresión para evaluar la carga de fallo por traccion diagonal en vigas de hormigón armado sin cercos. El estudio se fundamenta en resultados experimentales y en conceptos básicos de Mecánica de Fractura con el fin de analizar el efecto de tamaño. Se comprueba que la armadura longitudinal modifica el efecto de tamaño respecto al de elementos entallados sin armadura. Para cuantificar este efecto establecemos un modelo teórico que se contrasta con ensayos de vigas de hormigón armado. A partir del modelo propuesto se presenta una formulación para determinar la carga de fallo por tracción diagonal en vigas de hormigón sin cercos. Para facilitar la aplicación de la formulación se realizan una serie de simplificaciones y finalmente se obtiene una expresión en función de los parámetros convencionalmente utilizados en el diseño de estructuras de hormigón.

Palabras clave: Hormigón armado; fallo por tracción diagonal; efecto de tamaño; cortante en vigas sin cercos.

\section{ABSTRACT}

This paper proposes an expression to evaluate the load causing diagonal-tension failure of reinforced concrete beams without stirrups. The study is based on experimental tests results and basic concepts of Fracture Mechanics. It is observed that longitudinal reinforced bars modify the trend in size effect as compared to the one shown by un-reinforced notched beams. To quantify this effect, a theoretical model is established and validated against tests of reinforced concrete beams. From the proposed model, an expression is developed to determine the diagonal-tension strength in beams without stirrups. To facilitate the application in structural design, a series of simplifications has been done, so that the final expression is based on the parameters conventionally used in the design of concrete structures.

Keywords: Reinforced concrete; diagonal-tension failure; size effect; concrete shear.

(*) Universidad de Castilla-La Mancha, Ciudad Real (España).

Persona de contacto/Corresponding author: jacinto.rc@gmail.com (J. R. Carmona)

Cómo citar este artículo/Citation: Carmona, J. R., Ruiz, G. (2015). Evaluación de la carga de fallo por tracción diagonal en vigas de hormigón armado sin cercos a través del estudio del efecto de tamaño. Informes de la Construcción, 67(539): e109, doi: http://dx.doi. org/10.3989/ic.14.092.

Licencia / License: Salvo indicación contraria, todos los contenidos de la edición electrónica de Informes de la Construcción se distribuyen bajo una licencia de uso y distribución Creative Commons Reconocimiento no Comercial 3.o. España (cc-by-nc). 


\section{INTRODUCCIÓN}

La evaluación de la carga de fallo por tracción diagonal (fallo por cortante) en elementos de hormigón armado es un problema no resuelto de forma totalmente satisfactoria dentro del campo científico-tecnológico. El enorme interés suscitado por la cuestión objeto del presente estudio queda patente en los cientos de publicaciones realizadas sobre el tema en los últimos 50 años. A finales de los años 60 y como consecuencia de diversos fallos estructurales, como el de las vigas de unos almacenes de la Fuerzas aéreas en Ohio y Georgia (USA), se comenzaron a revisar las formulaciones existentes para estimar la carga de fallo por cortante, observándose como una posible causa de los fallos la existencia de un efecto de escala, es decir la dependencia de la resistencia del tamaño del elemento. En un principio este efecto se introdujo a partir de estudios estadísticos. A modo de ejemplo citamos los estudios de Zsutty (1) y posteriormente, entre otros, los estudios de Rebeitz (2) y Okamura and Higai (3). Simplificaciones de las expresiones empíricas como la propuesta por Zsutty se han incluido en normativas, por ejemplo en el antiguo Código Modelo (4) y en la normativa de hormigón en España, EHE-o8 (5) (6). La inclusión de estas formulaciones empíricas en códigos se debe principalmente a la sencillez en su aplicación y a que, en el rango de tamaños utilizado en construcción, han mostrado que los resultados obtenidos son suficientemente seguros para los tipos de hormigones frecuentemente utilizados hasta el momento.

A partir de mediados de los años 80 y con el fin de proporcionar una base teórica en las propuestas, se han desarrollado diversas teorías para determinar la carga de fallo, entre las que destacamos la denominada teoría del campo modificado de compresiones (7). En las formulaciones basadas en esta teoría, la resistencia a cortante en elementos de hormigón disminuye al aumentar la deformación en la armadura longitudinal (8) (9) (10) (11) (12) (13) (14). Si la deformación de estas barras la consideramos función del tamaño, el efecto de escala observado en los ensayos es reproducido de forma implícita por las formulaciones propuestas. En la bibliografía también encontramos otras metodologías que proporcionan expresiones a partir de modelos teóricos y que incluyen nuevos parámetros como la posición de la sección de fallo, la orientación de la misma, la resistencia a tracción del hormigón y el estado tensional dentro del elemento de hormigón (15) (16) (17) (18) (19 (20) (21) (22) (23).
Un nuevo punto de vista fue introducido por Reinhardt (24), el cual sugirió que las fórmulas de diseño para determinar la carga de fallo por cortante debían basarse en teorías basadas en Mecánica de la Fractura, dada la relación existente entre el progreso de las fisuras, el fallo del elemento y el efecto de escala que se pone de manifiesto en este tipo de fallo (25) (26) (27) (28) (29). La importancia de definir una formulación teórica que permita extrapolar entre resultados experimentales y elementos de construccion es fundamental, ya que el rango de tamaños en los ensayos realizados en laboratorio, y utilizados para calibrar formulaciones, suele ser inferior generalmente a los utilizados en construcción. El análisis del efecto de escala dentro del ámbito de la Mecánica de la Fractura nos permite la obtención de expresiones que permiten la extrapolacion de resultados y su análisis asintótico. Diversos investigadores han realizado propuestas de expresiones para evaluar la carga de fallo por tracción diagonal introduciendo conceptos de Mecánica de la Fractura, así podemos mencionar los trabajos de Bažant y Sun (30), Bažant y Yu (31) (32) y Kim y Park (33).

En el presente trabajo, a partir de un modelo teórico basado en observaciones experimentales y conceptos de Mecánica de la Fractura, se proponen dos expresiones para evaluar la carga de fallo por tracción diagonal, según las consideraciones que se realizan sobre el comportamiento asintótico del efecto de tamaño o efecto de escala. Se considera que el fallo por tracción diagonal se produce de forma frágil por el crecimiento inestable de una fisura que nace en una zona entre el punto de aplicación de la carga y el apoyo (27) (28). En la Figura 1 mostramos el mapa de fisuración típico en una rotura por tracción diagonal (cortante), remarcando la fisura en la que se produce la inestabilidad, y en Figura $1 \mathrm{~b}$ la curva carga-desplazamiento en el punto de aplicación de la carga típica de un ensayo de una viga de hormigón armado sin cercos que rompe por cortante. Se aprecia cómo tras alcanzar la carga máxima se produce un fallo abrupto del elemento de hormigón armado. Las nuevas expresiones que se desarrollan en el artículo han sido calibradas mediante una base de datos de ensayos de vigas de hormigón (29) (34) (35) (36) (37) (38) (39) (40) (41) (42) (43) (44) (45) (46) (47) (48) (49) (50) (51) (52) (53) (54). Finalmente se propone una expresión simplificada, con un carácter eminentemente práctico, que permite evaluar la carga de fallo por tracción diagonal. a)

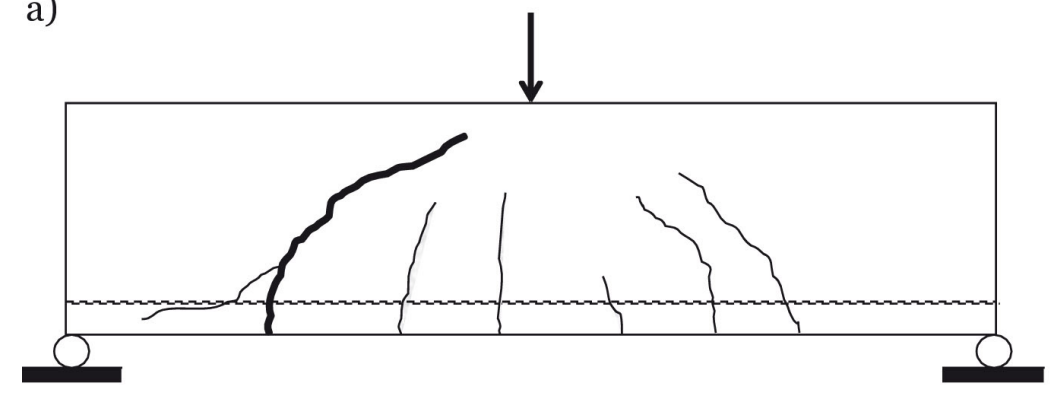

b)

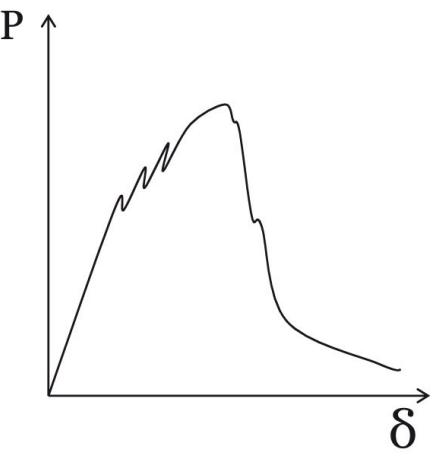

Figura 1. (a) Mapa de fisuración típico de una rotura por tracción diagonal (cortante) en una viga de hormigón armado sin cercos; (b) curva típica carga-desplazamiento de un ensayo a tres puntos de una viga de hormigón armada sin cercos. 


\section{RESULTADOS EXPERIMENTALES Y FUNDAMENTO TEÓRICO}

Con el fin de analizar la influencia del tamaño del elemento y de la cuantía de armado, se planteó una campaña experimental para estudiar la propagación de fisuras de cortante en elementos de hormigón armado. La geometría seleccionada para realizar los ensayos es una viga con una entalla asimétrica situada a un canto del apoyo, Figuras 2a y 2b. La entalla se introdujo con el fin de simular una fisura que se ha propagado durante su fase inicial y de evitar la aparición de mapas complejos de fisuración. De esta forma se pretendía estudiar la relación directa entre la propagación de fisuras y el fallo. Los resultados de la campaña están detallados en la referencia (55). En la campaña experimental se observó que el fallo por tracción diagonal se produce cuando una fisura de cortante ha descrito una cierta trayectoria y el armado longitudinal es incapaz de controlar el progreso de esa fisura.

La influencia de la cuantía durante el proceso de fisuración es mostrada en la Figura 2c. En ella se representa en abscisas la cuantía de armado, $\rho$, y en ordenadas la carga para la que se localiza el daño en la entalla, $P_{c}$, de forma adimensionalizada. La presencia de una armadura da lugar a una hiper-resistencia en la carga resistida por las vigas en el momento en el que se localiza el daño en la entalla, es decir el momento en el que una fisura aparece y comienza a propagarse a partir de la punta de la entalla. El ajuste lineal mostrado en la Figura 2c representa de forma bastante aproximada la tendencia experimental observada, siendo el coeficiente de correlación de Pearson, $R$, próximo a la unidad. El efecto de tamaño en los ensayos es mostrado en la Figura 2d. En este gráfico representamos la carga a la que se produce la localización del daño en la entalla, frente al tamaño del elemento, ambos de forma adimensionalizada. Incluimos los resultados para vigas sin ningún tipo de armado. Estos elementos sin armado al encontrarse entallados son susceptibles de ser analizados con la ley de efecto de escala de Bažant (56), la cual mostramos con una línea continua para los resultados obtenidos en la Figura 2d y se define como:

$$
\frac{P_{c}}{b h f_{t}}=\frac{B_{o}}{\sqrt{1+\frac{\beta_{H}}{\beta_{o}}}}
$$

Donde, $P_{c}$ es la carga para la cual se produce la propagación de la fisura en la punta de la entalla y $\beta_{H}$ es el denominado número de fragilidad de Hillerborg (57), el cual se define como el cociente entre el tamaño del elemento, representado en este caso por el canto $h$, y la longitud característica del material, $l_{c h}(58)$.

$$
\beta_{H}=\frac{h}{l_{c h}}, \text { donde } l_{c h}=\frac{E_{c} G_{F}}{f_{t}^{2}}
$$

$E_{c}$ es el módulo de elasticidad del hormigón, $G_{F}$ es la energía de fractura y $f_{t}$ es la resistencia a tracción. $B_{o}$ y $\beta_{o}$ son dos constantes adimensionales independientes del tamaño del elemento. Estas constantes son determinadas a partir de los resultados experimentales de las vigas sin ningún tipo de armado. En nuestro caso toman los valores o,19 y 1,45 respectivamente. Las constantes son válidas para la geometría elegida y el material empleado es estos ensayos. Cuando éstos varían, las constantes cambian. El efecto de escala observado en vigas armadas prácticamente desaparece con una cuantía longitudinal del 0,13\%, y para el o,26 \% el efecto de tamaño es muy tenue. Así pues, la carga de propagación en las vigas armadas no parece tender hacia una resistencia nula al aumentar el tamaño, sino hacia una resistencia residual, función de la cuantía de armado. Para facilitar las comparaciones se han dibujado unas líneas de interpolación con trazo discontinuo. a)

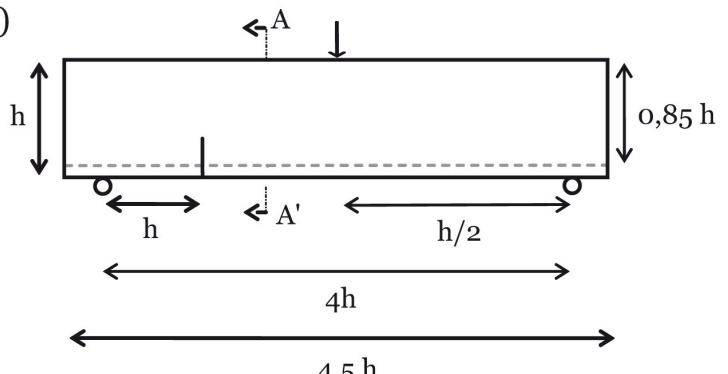

b)

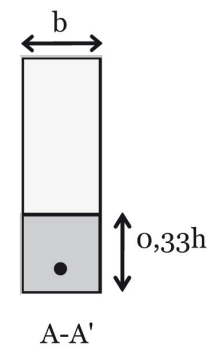

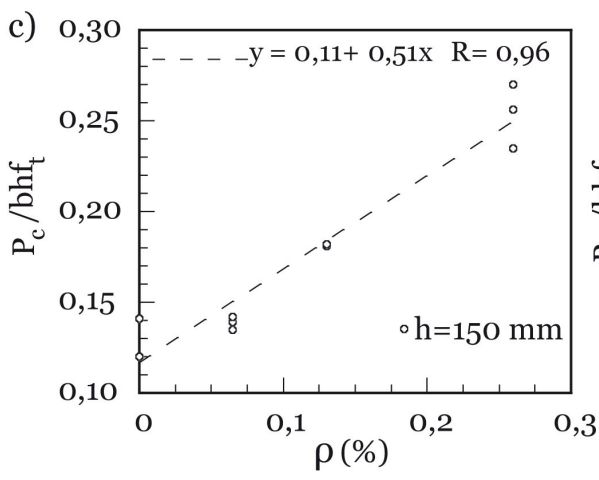

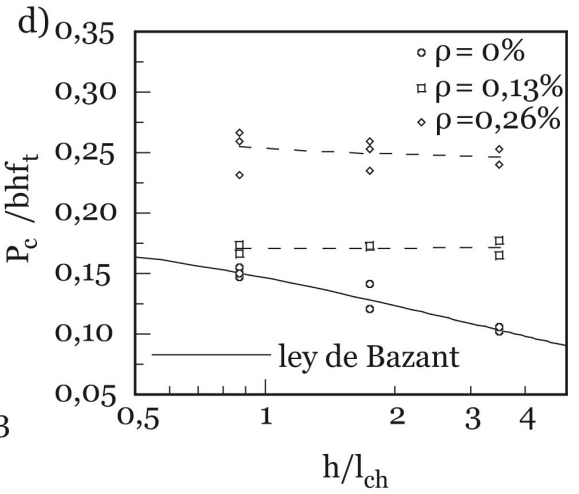

Figura 2. (a) Geometría de la viga ensayada; (b) sección A-A'; (c) Influencia de la cuantía de armado en la carga de fisuración para vigas de $150 \mathrm{~mm}$ de canto;(d) Influencia del tamaño en la carga de fisuración. 
Según Ruiz (59), la carga durante un proceso de fractura en un elemento de hormigón armado se puede representar en función de la geometría del elemento, de las condiciones de contorno y de las propiedades de los materiales. Esta carga se puede descomponer de forma sencilla en dos términos. El primero representará la capacidad resistente del hormigón, $\sigma$, y el segundo la hiper-resistencia debida a la presencia del armado, $\sigma_{\Delta}$. Las armaduras provocan una resistencia adicional al progreso de las fisuras, el cual se puede superponer a la resistencia que ofrece el hormigón. Así pues podemos escribir:

$$
\sigma_{N}=\sigma_{C}+\sigma_{\Delta}
$$

$\sigma_{N}$ se define como la tensión nominal que resiste el elemento, $\sigma_{N}=P /$ Área. Esta tensión nominal es un parámetro relacionado con la carga que es soportada por el elemento de hormigón, tal y como se desprende de su definición, pero no tiene relación directa con las tensiones normales. La capacidad del hormigón se representa por un término, $\sigma_{c}$, que tiene en cuenta el efecto de escala. En este trabajo se han seleccionado dos expresiones para $\sigma_{c}$, una basada en la Mecánica de la Fractura Elástica Lineal (LEFM) cuya aplicación al estudio de la carga máxima fue propuesta por Reinhardt (24), y una segunda opción mediante la ley de efecto de escala de Bažant (SEL) (58) para materiales de comportamiento cuasifrágil, que ya ha sido utilizada en el análisis mostrado en la Figura 2. Dado que existe semejanza entre los patrones de fisuración para varios tamaños de viga (57), se pueden aplicar las formulaciones expuestas para analizar el efecto de escala.
La hiper-resistencia debida a la presencia del acero se representa a través de una relación lineal entre dicha hiper-resistencia, $\sigma_{\Delta}$, y la cuantía de armado, $\rho$, de acuerdo con las observaciones experimentales. Así pues, tenemos dos expresiones con un tratamiento asintótico del efecto de escala diferente para determinar la tensión nominal durante el proceso de fisuración.

$$
\begin{gathered}
\sigma_{N, L E F M}=K_{0} \beta_{H}^{-1 / 2} f_{t}+K_{\Delta} \rho f_{u} \\
\sigma_{N, S E L}=\frac{B_{0} f_{t}}{\sqrt{1+\frac{\beta_{H}}{\beta_{o}}}}+K_{\Delta} \rho f_{y}
\end{gathered}
$$

En ambos casos la carga depende de la resistencia a tracción, de la cuantía de armado y de la resistencia del acero, y además del tamaño intrínseco del elemento. $\beta_{h}, K_{o}, K_{\Delta}, B_{o}$ y $\beta_{o}$ son unas constantes adimensionales independientes del tamaño para vigas escaladas entre sí. A partir de los datos de la campaña experimental podemos evaluar estos coeficientes de forma gráfica. Se considera que la tensión en el acero va a ser constante en el momento del fallo para elementos homotéticos, por lo que la tensión puede ser representada por el límite elástico multiplicado por una constante que queda incluida en $K_{\Delta}$. La ecuación [4] se linealiza y, a partir de los datos experimentales, se realiza una regresión lineal obteniéndose el valor de las constantes $K_{o}$ y $K_{\Delta}$. La metodología seguida es semejante a la que usan Bazant y Yu en (32).

En la Figura 3a se muestran las regresiones realizadas, utilizando la expresión [4] para determinar las constantes $K_{o} \mathrm{y}$ a)

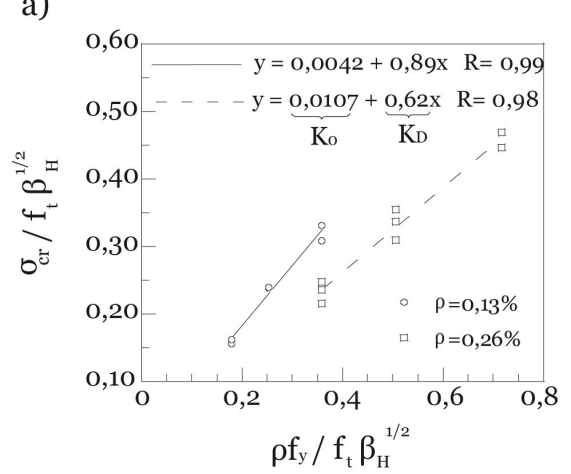

c)

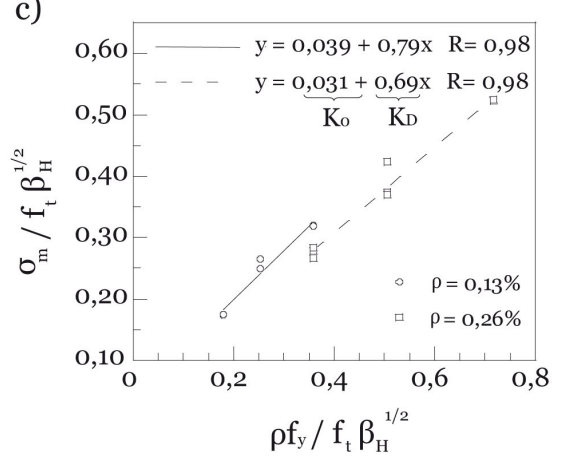

b)

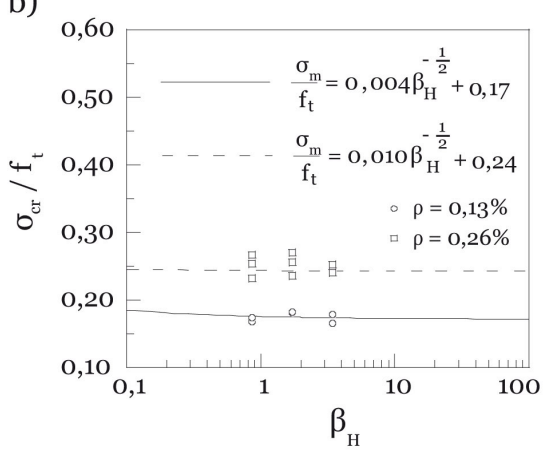

d)

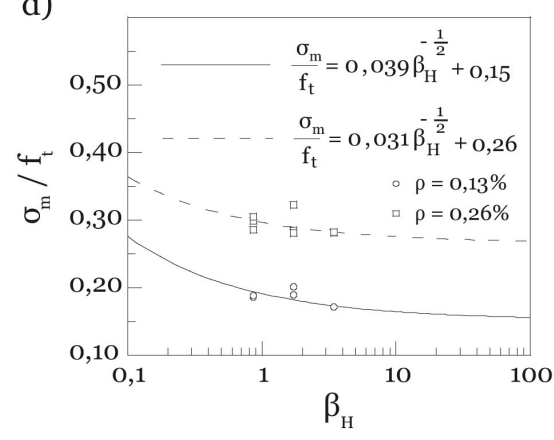

Figura 3. (a) Resultados de la regresión para calibrar $\mathrm{K}_{\mathrm{o}} \mathrm{y} \mathrm{K}_{\Delta}$ para la carga de inicio de la propagación de la fisura en la entalla; (b) efecto de tamaño en la carga de inicio de la propagación de la fisura en la entalla; (c) resultados de la regresión para calibrar $\mathrm{K}_{\mathrm{o}}$ y $\mathrm{K}_{\Delta}$ para la carga máxima; (d) efecto de tamaño en la carga máxima. 
$K_{\Delta}$ para las cargas en las que se detecta el inicio de la propagación de la fisura en la entalla. También se muestra el valor del coeficiente de correlación de Pearson, $R$, obtenido. La ley obtenida se compara con los resultados experimentales en la Figura 3b. El efecto de tamaño tiende a desaparecer cuando el tamaño de la viga tiende a infinito, véase la Figura $3 \mathrm{~b}$. La tensión nominal converge a un valor diferente de cero, función de la cuantía de armado. El efecto de escala debido a la presencia de armado se atenúa frente al que tendrían elementos sin armadura, tal y como se observó experimentalmente.

En elementos de hormigón armado, las fisuras producen un efecto similar al de la entalla introducida en los ensayos. Si consideramos que el fallo por tracción diagonal se produce después de un desarrollo del proceso de fisuración y no en su iniciación, y que los mapas de fisuración en el momento del fallo son similares al variar el tamaño, la ecuación [4] puede representar la carga máxima o carga de fallo por tracción diagonal. La utilización de la Mecánica de Fractura Elástica Lineal es considerada como suficientemente aproximada en este caso, debido a que el fallo por tracción diagonal es predominantemente frágil (20). La Figura $3 \mathrm{c}$ muestra la regresión lineal realizada para determinar las constantes $K_{o}$ y $K_{\Delta}$ para las cargas máximas (carga de fallo por tracción diagonal) en las vigas ensayadas. En la Figura 3d, la ley de efecto de tamaño obtenida se compara con los resultados experimentales. Se observa que la expresión propuesta capta la tendencia observada en los resultados experimentales. El análisis se ha realizado con la ecuación [4] dado que tenía un número de parametros libres menor y el ajuste era menos favorable que en el caso de la ecuación [5].

\section{EVALUACION DE LA RESISTENCIA A TRACCIÓN DIAGONAL EN VIGAS SIN CERCOS}

Tal y como se ha descrito en el apartado anterior, la carga de fallo de un elemento de hormigón con armadura longitudinal en la parte traccionada se puede expresar como la suma de dos términos, uno que representa la propia resistencia del hormigón al avance de la fisura y otro que indica el aumento de resistencia debido a la existencia del armado longitudinal. A partir de las ecuaciones [4] y [5] y con el fin de obtener una expresión que permita evaluar la carga de fallo por tracción diagonal de un elemento de hormigón, se fijan una serie de parámetros libres, que representen las constantes en las ecuaciones citadas, con el fin de realizar un ajuste estadístico a partir de resultados de ensayos.

En el caso de la expresión donde el comportamiento del hormigón se modela usando la Mecánica de la Fractura Elástica Lineal (LEFM), ecuación [4], la expresión a calibrar queda de la siguiente forma:

$$
\sigma_{N, L E F M}=K_{0} \lambda^{m}(\rho+1)^{n} \beta_{H}^{-1 / 2} f_{t}+K_{\Delta} \lambda^{r} \rho^{s} f_{y}
$$

Donde $\lambda$ es la esbeltez de la viga, calculada como el cociente entre la distancia desde el punto de aplicación de la carga al apoyo (vigas biapoyadas) y el canto del elemento. En la sección 2 del artículo no se tuvo en cuenta la esbeltez dado que era un parámetro constante en la campaña experimental. Debido a que los patrones de fisuración sí son dependientes de la esbeltez, hemos estimado necesario introducir ésta como parámetro en la calibración. La segunda expresión (SEL) obtenida a partir de la ecuación [5], queda como:

$$
\sigma_{N, S E L}=\frac{B_{0} \lambda^{m}(\rho+1)^{n} f_{t}}{\sqrt{1+\frac{\beta_{H}}{\beta_{0} \lambda^{p}(\rho+1)^{q}}}}+K_{\Delta} \lambda^{r} \rho^{s} f_{y}
$$

En estas expresiones $K_{o}, K_{\Delta}, B_{o}, \beta_{o}, m, n, p, q, r$ y $s$ son parámetros libres de valor constante. La principal diferencia entre ambas expresiones la encontramos en la representación del efecto de escala. El primer término en las ecuaciones [6] y [7] no debería, teóricamente, estar afectado por la cuantía de armado. No obstante, se ha introducido en las constantes un parámetro dependiente de la cuantía y cercano a la unidad, $\rho+1$, para tener en cuenta los cambios que las cuantías elevadas se producen en los mapas de fisuración al variar el tamaño (6o) (61).

Para la formulación basada en la Mecánica de Fractura Elástica Lineal en tamaños grandes, el valor del efecto de escala, es una recta de pendiente $-1 / 2$ (51), cuando se representa la dependencia de la resistencia frente al tamaño en un gráfico con escala logarítmica en abscisas y ordenadas. Para la Formulación SEL el efecto de escala desaparece, tendiendo a o para elementos de tamaño pequeño. En todo caso, los efectos de escala representados se encuentran entre $-1 / 2$, que es la pendiente indicada por la teoría de la Mecánica de la Fractura Elástica Lineal, y o, que indicaría la ausencia de efecto de escala, teoría de la plasticidad. Este hecho es de gran importancia, ya que numerosas formulaciones propuestas, al estar basadas en criterios empíricos u otras teorías, no cumplen estas limitaciones, obteniéndose resultados para el efecto de efecto de escala incompatibles con la termodinámica de los medios continuos.

Con el propósito de calibrar el valor de las constantes se va a realizar un ajuste estadístico. Hemos usado una base con los datos de 401 ensayos de vigas de hormigón armado sin cercos (29) (34) (35) (36) (37) (38) (39) (40) (41) (42) (43) (44) (45) (46) (47) (48) (49) (50) (51) (52) (53) (54). Los datos analizados están a la disposición de los lectores solicitándolos a la dirección de cualquiera de los autores del artículo. En la Figura 4 se representan los resultados contenidos en la base de datos según diferentes variables que caracterizan su geometría y materiales. En ordenadas se representa en todas las gráficas la tensión nominal de rotura, definida como la carga máxima soportada por la viga durante su ensayo dividida del área de la sección. En abscisas se representa: el canto, la cuantía de acero, el tamaño máximo de árido, la esbeltez de la viga (cociente entre la distancia desde la carga al apoyo y el canto de la viga) la resistencia del acero y la resistencia a compresión del hormigón.

En la Figura 4a se observa cómo el 95 \% de los ensayos están realizados en vigas entre $100 \mathrm{~mm}$ y $900 \mathrm{~mm}$, siendo el canto medio aproximadamente de $350 \mathrm{~mm}$. En construcción es normal encontrar vigas con cantos sensiblemente superiores. De forma general se observa que al aumentar el canto disminuye la resistencia, Figura 4a, y al aumentar la cuantía aumenta la resistencia, Figura 4b. En la Figura 4d se observa que la esbeltez de las vigas tiene un valor medio en los ensayos entre 2 y 3 , siendo frecuente alcanzar valores muy superiores en la ejecución de losas o vigas de hormigón armado. De forma más tenue también se observa la tendencia de que al aumentar la resistencia del hormigón existe un aumento de la resistencia a la tracción diagonal, Figura 4f. Los resultados para el tamaño 
de árido, la esbeltez y la resistencia del acero no indican una tendencia clara. En la base de datos se ha observado que los recubrimientos varían entre el 5 y el $10 \%$ del canto de la viga. Así pues, dada la baja variabilidad de este parámetro no se ha incluido en la formulación, con el fin de facilitar el análisis.

Un problema que presentan algunas series de resultados experimentales consultadas es la falta de caracterización de los materiales utilizados en la ejecución de las vigas, siendo frecuente en el caso del hormigón que únicamente se caracterice éste a través de la resistencia a compresión. En orden a obtener el resto de propiedades no incluidas en la caracterización realizada en los ensayos y necesaria para la formulación, hemos utilizado las expresiones del Código Modelo (4).

Todos los coeficientes libres de las constantes adimensionales han sido calibrados mediante un ajuste estadístico. La función objetivo seleccionada para minimizar el cuadrado del error de la regresión $\left(s_{L}^{2}\right)$ ha sido:

$$
s_{L}^{2}=\frac{1}{n-n_{p}} \sum_{i=1}^{n}\left(\ln \frac{v_{i}^{*}}{v_{i}}\right)
$$

Donde $n_{p}$ es el número de parámetros libres en el ajuste, $v_{i}^{*}$ representa a cada uno de los resultados de la resistencia de los ensayos incluidos en la base de datos y $v_{i}$ es el valor de la resistencia a cortante según la formulación propuesta. El objeto de esta función objetivo es ponderar los valores de los ensayos con cantos menores, debido a que el coeficiente de variación de los ensayos no es constante en el rango de los tamaños de las vigas ensayadas. Esto provoca que los ajustes lineales de mínimos cuadrados resulten sesgados, ya que consideran el rango de variación de los datos constante (32). Los coeficientes son evaluados de tal forma que el error anteriormente definido sea mínimo. La minimización ha sido llevada a cabo mediante una subrutina programada en el lenguaje de programación GAMS. El coeficiente de variación $\omega$ queda definido por la siguiente expresión.

$$
\omega=\left(e^{s_{L}}-e^{-s_{L}}\right) / 2
$$

Los valores finales de los coeficientes obtenidos para los parámetros libres se muestran en la Tabla 1. Hemos estimado estos parámetros tanto para la formulación basada en Mecánica de Fractura Elástica Lineal (LEFM) como para la que representa el efecto de escala mediante la ley de Bažant (SEL). a)
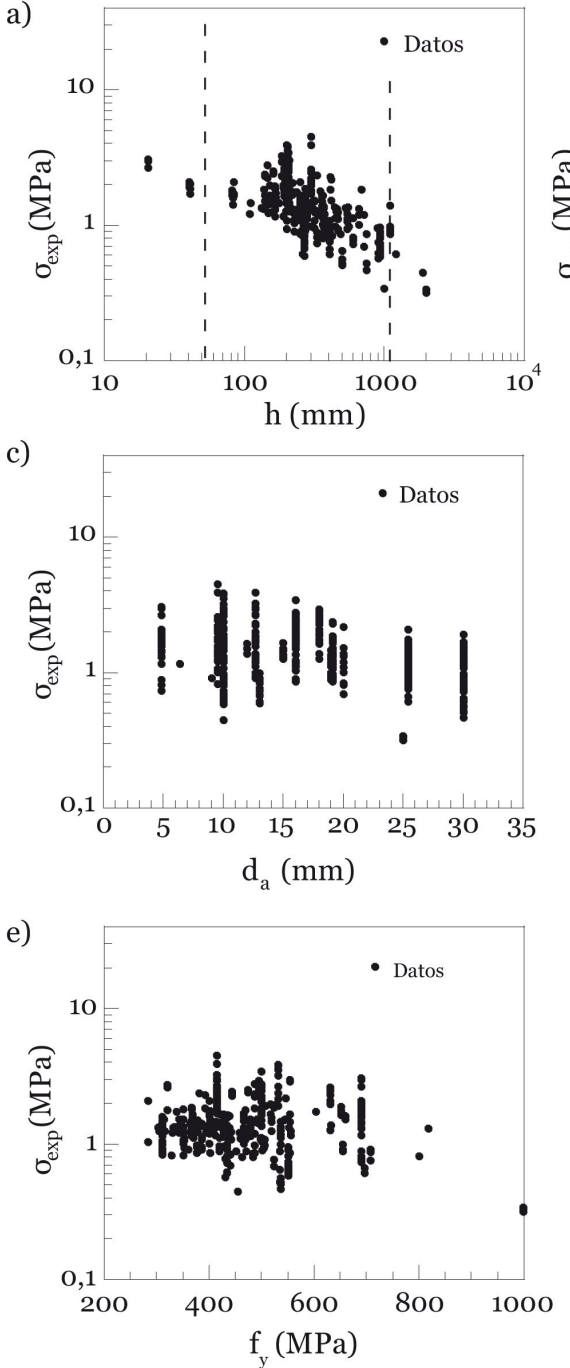

b)

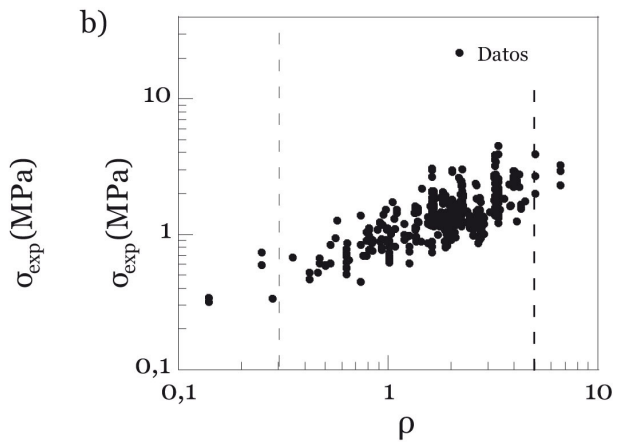

d)

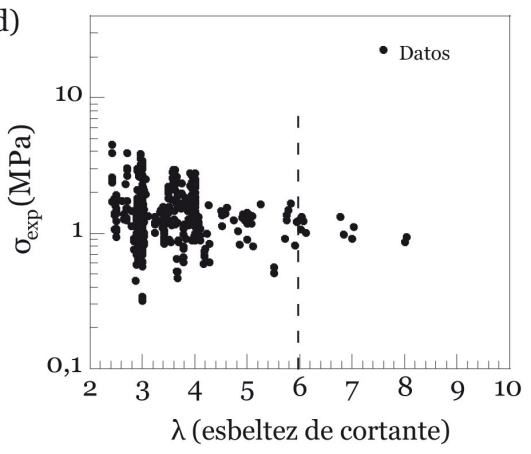

f)

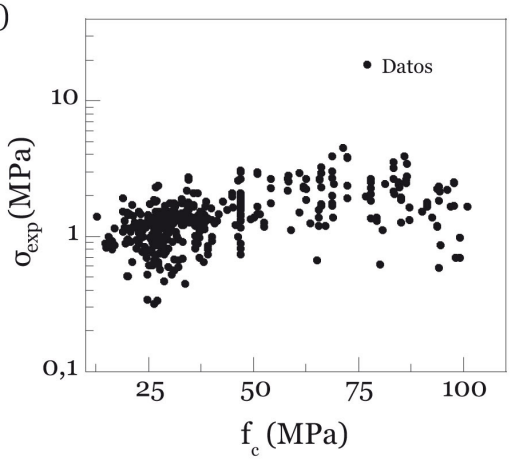

Figura 4. Base de datos. Influencia en la carga máxima debida a: (a) canto de elemento, h; (b) cuantía de armado, $\rho$; (c) tamaño máximo del árido, $\mathrm{d}_{\mathrm{a}}$; (d) esbeltez del elemento, $\lambda$; (e) límite elástico del acero, $\mathrm{f}_{\mathrm{y}}$; (f) resistencia a compresión del hormigón, $\mathrm{f}_{\mathrm{c}}$. 
Tabla 1. Valores de los coeficientes libres en las formulaciones propuestas.

\begin{tabular}{|l|c|c|}
\cline { 2 - 3 } \multicolumn{1}{c|}{} & $\boldsymbol{\sigma}_{\mathrm{N}, \mathrm{LEFM}}$ & $\boldsymbol{\sigma}_{\mathrm{N}, \mathrm{SEL}}$ \\
\hline $\mathrm{K}_{\mathrm{o}}$ & 0,7 & - \\
\hline $\mathrm{B}_{\mathrm{o}}$ & - & 14,5 \\
\hline$\beta_{\mathrm{o}}$ & - & $1,58 \cdot 10^{-3}$ \\
\hline $\mathrm{K}_{\Delta}$ & $3,86 \cdot 10^{-4}$ & $3,02 \cdot 10^{-4}$ \\
\hline $\mathrm{m}$ & $-1,22$ & $-1,47$ \\
\hline $\mathrm{n}$ & 0,48 & $-0,9$ \\
\hline $\mathrm{p}$ & - & 0,64 \\
\hline $\mathrm{q}$ & - & 3,29 \\
\hline $\mathrm{r}$ & 0,37 & 0,5 \\
\hline $\mathrm{s}$ & 0,9 & 0,9 \\
\hline $\mathrm{s}_{\mathrm{L}}{ }^{2}$ & 0,033 & 0,030 \\
\hline$\omega$ & 0,18 & 0,19 \\
\hline
\end{tabular}

a)

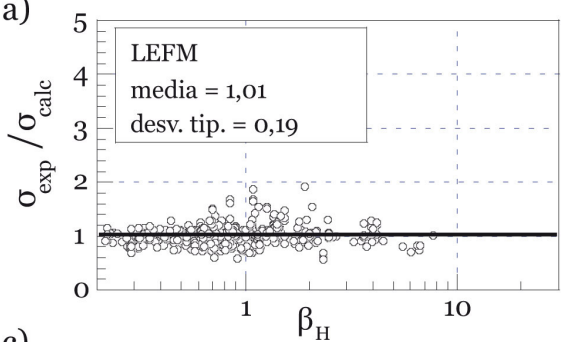

c)
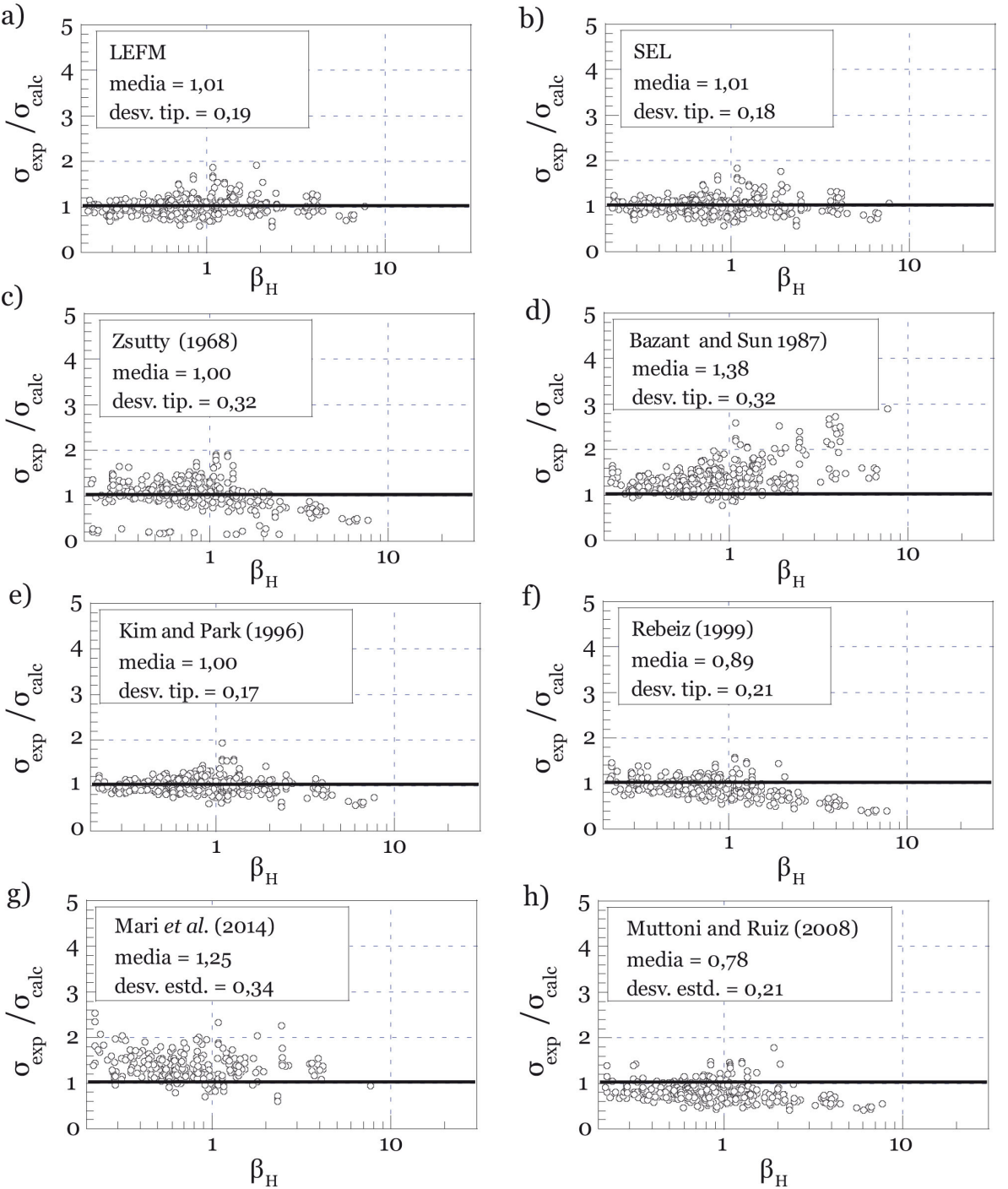

Figura 5. Comparación entre resultados experimentales y diferentes formulaciones:

(a) LEFM, formulación basada en Mecánica de la Fractura Elástica Lineal;

(b) SEL, formulación basada en el efecto de tamaño de Bažant; (c) Zsutty (1968);

(d) Bažant y Sun (1987); (e) Kim y Park (1996); (f) Rebeiz (1999); (g) Marí et al. (2014);

(h) Muttoni y Ruiz (2008). $\beta_{\mathrm{H}}$ es el denominado número de fragilidad de Hillerborg [Ec. 2], que relaciona el tamaño del elemento y las características mecánicas del hormigón. 
Se observa que la media de los resultados del cociente entre los datos de los ensayos y los resultados evaluados con las expresiones presenta valores cercanos a la unidad. Los resultados de las formulaciones propuestas (LEFM y SEL) conjuntamente con la de Kim y Park muestran resultados bastante similares. La formulación propuesta por Zsutty, si bien obtiene un valor medio cercano a uno, presenta una desviación alta tanto para para tamaños pequeños como grandes respecto a la media. Con la formulación propuesta por Bažant y Sun se obtienen en general valores por debajo de la media, al igual que sucede con la de Marí y colaboradores, es posible que ambas formulaciones tengan introducidos algunos coeficientes de seguridad en su desarrollo que justifiquen esta desviacion de la media. Se observa que la propuesta de Rebeiz no ajusta bien los valores para tamaños grandes. Por último, la expresión propuesta por Muttoni y Ruiz, basada en la teoria del Campo Modificado de Compresiones, ajusta con valores de resistencia superiores a la media de los ensayos, con una desviación del orden de la evaluada en las expresiones propuestas. Para valores altos del tamaño se observa una desviacion de los resultados respecto a la media.
En la Figura 6 se representan los resultados obtenidos con las formulaciones LEFM y SEL, en escala logarítmica, para diferentes valores de la resistencia a compresión del hormigón y del tamaño intrínseco del elemento, $\beta_{H}$. En cada una de las gráficas se muestra en abscisas el tamaño del elemento y en ordenadas la resistencia. En cada fila se modifica la resistencia del hormigón. La primera columna muestra resultados con la formulación LEFM y la segunda con la formulación SEL. Dentro de cada gráfica se dibujan curvas para distintas cuantías. Observamos como para las cuantías más elevadas la resistencia es mayor y el efecto de escala prácticamente desaparece.

En las gráficas incluidas en la Figura 6 se dibujan unas líneas verticales discontinuas indicando el rango de tamaños que encontramos en la mayoría de los casos que se presentan en el diseño de estructuras de hormigón. Este rango vendría representado por un número de fragilidad de Hillerborg de entre 0,2 y 30 . Si tomamos como promedio que la longitud característica de un hormigón convencional puede estar comprendida entre $150 \mathrm{~mm}$ y $500 \mathrm{~mm}$, las líneas indican vigas de entre $100 \mathrm{~mm}$ y $3000 \mathrm{~mm}$ de canto. En ese rango se pro-
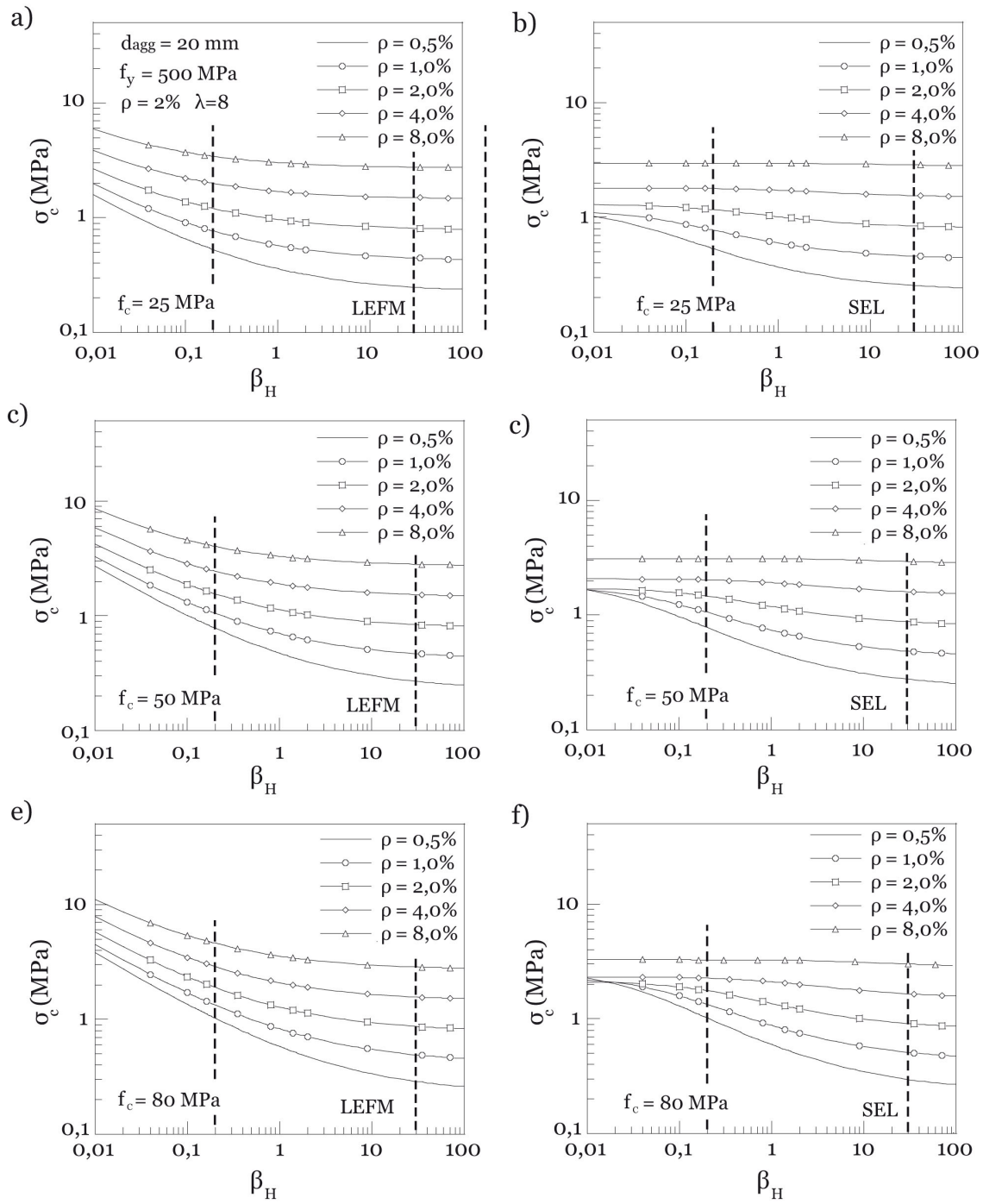

Figura 6. Representación de la tensión nominal de rotura frente al tamaño del elemento para una viga de esbeltez 8 con una cuantía del $2 \%$ y un tamaño máximo de árido de $20 \mathrm{~mm}$ :

(a) LEFM, fc = $25 \mathrm{MPa}$; (b) SEL, fc = $25 \mathrm{MPa}$; (c) LEFM, fc = $50 \mathrm{MPa}$; (d) SEL, fc = 50 MPa; (e) LEFM, fc = $80 \mathrm{MPa}$; (f) SEL, fc $=80 \mathrm{MPa}$. 
duce un efecto de escala acusado, sobre todo para cuantías medias y bajas. En la formulación SEL se observa que para cuantías hasta del $2 \%$, en tamaños pequeños la resistencia tiende hacia el mismo valor. Esta observación coincide con la mostrada en la referencia (62). Para tamaños muy pequeños, la apertura de fisura, cuando ésta alcanza una profundidad que provoca la inestabilidad, es muy pequeña y no se logra movilizar la armadura, por lo que la resistencia únicamente dependería del término del hormigón. Conforme crece el tamaño el término del hormigón decrece y el término del acero va teniendo mayor importancia.

\section{FORMULACIÓN SIMPLIFICADA}

Con el fin de establecer una formulación de carácter práctico para evaluar la carga de fallo en elementos de hormigón armado sin cercos, se van a realizar una serie de simplificaciones en las formulaciones propuestas indicadas en el apartado anterior. En primer lugar, limitamos el rango de aplicación de la formulación a tamaños representados por el número de fragilidad de Hillerborg de entre 0,2 y 30, abarcando la mayoría de los casos que se presentan en el diseño de estructuras de hormigón. Asimismo, dadas las características de los ensayos que sirvieron para la calibración, restringimos el uso de la expresión que se propone a hormigones ordinarios, armados con aceros de entre 400-500 $\mathrm{MPa}$ de límite elástico.

En la Figura 6 se observa que cada una de las curvas obtenidas puede ser representada por una recta que una los puntos de corte de la curva con los límites establecidos, sin que el error cometido sea importante. La representación de una línea recta en escala bilogaritmica corresponde a una expresión del tipo:

$$
\sigma_{N}=A \beta_{H}^{-\alpha}
$$

Donde $A$ representa el punto de corte con el eje de ordenadas en la abcisa unidad y $\alpha$ representa la pendiente de la recta en escala bilogarítmica. Para hallar los coeficientes $A$ y $\alpha$, realizamos un ajuste con los valores obtenidos con las formulaciones en los límites establecidos. El coeficiente $A$ dependera de la esbeltez, resistencia del hormigón y cuantía, y el coeficiente $b$ de la cuantía, tal y como se desprende de la observacion de la Figura 6. La formulación obtenida tras el ajuste es:

$$
\begin{aligned}
& \frac{V_{m}}{b d}=\Phi(100 \rho)^{0,6} f_{c k}^{0,3}\left(\frac{d}{d_{0}} f_{c k}^{0,7}\right)^{-\frac{0,15}{100+0,3}} \\
& \text { si } \lambda_{c}<8 \quad \Phi=0,37-0,0125 \lambda \\
& \text { si } \lambda_{c} \geq 8 \quad \Phi=0,27
\end{aligned}
$$

Donde $V_{m}$ es el cortante, $\rho$ es la cuantía de armado, $d$ es el canto útil (canto total menos recubrimiento), $b$ es el ancho de la sección, $f_{c k}$, es la resistencia a compresión del hormigón en MPa y $\lambda_{c}$ es la esbeltez a cortante, definida como la distancia desde el punto de momento flector máximo al punto de momento flector nulo dividida del canto del elemento. $d_{o}$ es una constante de valor $3800 \mathrm{~mm}$. La ecuación [11] es dimensional, es decir, hay que introducir la resistencia del hormigón en $\mathrm{MPa}$, el canto en mm y la cuantía en tanto por uno para obtener el resutado de la resistencia en MPa. Para una cuantía infinita se obtendría un valor del exponente $\alpha$, véase la ecuacion [10], igual a o, es decir, desaparecería el efecto de escala. Teniedo en cuenta la dispersión de los resultados en la calibración, para hallar el valor característico de la resistencia a cortante se introduce un coeficiente evaluado a partir de la desviacion estandar (o desviacion típica), según el dato expuesto en la Figura 5b. Este coeficiente, cuyo valor se ha redondeado a 0,7 , resulta de restar a la media, la desviación tipica multiplicada por 1,64, de forma que el valor obtenido tiene una probabilidad del $95 \%$ de ser superado. Asimismo, para determinar el valor de cálculo, se introduce un coeficiente parcial de seguridad, $\gamma_{c}$ que se ha tomado igual a 1,5 en situación persistente o transitoria. Teniendo en cuenta los coeficientes introducidos se obtiene un índice de fiabilidad en la expresión de 3.1. La expresión para cálculo quedaría finalmente como:

$$
\begin{gathered}
\frac{V_{d}}{b d}=\frac{0,7 \Phi(100 \rho)^{0,6} f_{c k}^{0,3}}{\gamma_{c}}\left(\frac{d}{d_{0}} f_{c k}^{0,7}\right)^{-\frac{0,15}{100 \rho+0,3}} \\
\left\{\begin{array}{lc}
\text { si } \quad \lambda_{c}<8, & \Phi=0,37-0,0125 \lambda \\
\text { si } \quad \lambda_{c} \geq 8, & \Phi=0,27
\end{array}\right.
\end{gathered}
$$

La comparación directa entre normativas no se puede realizar, ya que los párametros de los que dependen las expresiones no están evaluados con los mismos requisitos de seguridad para los materiales (64). No obstante, esta comparación entre ellas puede aportar una idea sobre los valores de resistencia y su variacion con el tamaño y cuantía. En la Figura 7 se comparan diferentes resultados de la ecuaciones [11] y [12], con diferentes normativas de aplicación actuales, ACI (63), EC-2 (65), EHE-o8 (5) y nivel 1 del nuevo Código Modelo (14). En abcisas se muestra el canto útil del elemento de hormigon armado y en ordenadas la resistencia a cortante (fallo por tracción diagonal). Las características de la sección para la que se ha evaluado la resistencia se muestran en la propia figura.

Observamos como para cuantías bajas y resistencias bajas de armado los resultados obtenidos con la ecuación [11], son similares a los resultados del EC-2 y EHE-08, quedando los resultados obtenidos con el Código ACI por encima. Los resultados para la propuesta cuando introducimos factores de seguridad están en el orden de los que se obtienen con la expresión del Código Modelo. El aumento de resistencia tiene una mayor influencia en las expresiones de las normativas que en las propuestas realizadas. $\mathrm{Al}$ aumentar la cuantía aumenta en mayor medida la tensión de fallo en las propuestas quedando éstas en el orden de magnitud de la tensión establecida por el ACI, el EC-2 y la EHE-o8 y quedando por encima de la indicada por Código Modelo. En la expresion simplificada se ha incluido la influencia de la esbeltez, parámetro no recogido por las normativas que hemos utilizado en la comparación.

El procedimiento seguido en la introducción de la seguridad es sencillo, y estimamos que su virtud es, sobre todo, mostrar las diferencias entre expresiones obtenidas con valores medios y aquellas en las que se han introducido criterios de seguridad. Aparentemente la formulación aplicada a cálculo quedaría del lado de la seguridad, al comparar con otras obtenidas para valores medios. La dispersión respecto a la me- 
a)

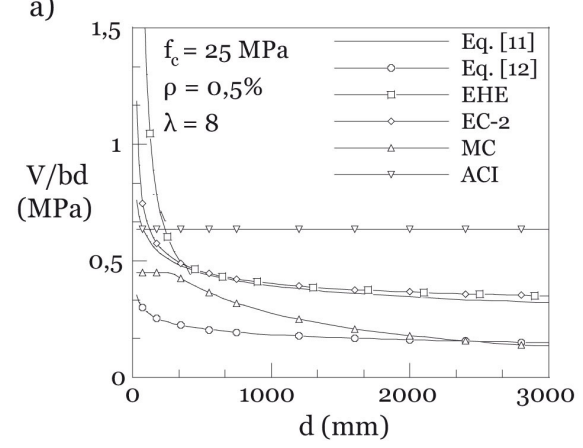

c)

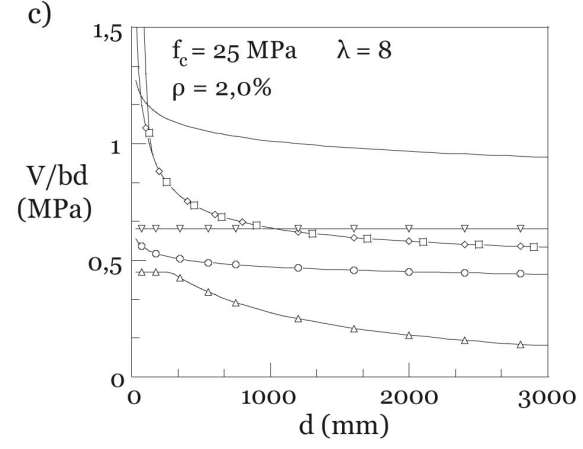

b)

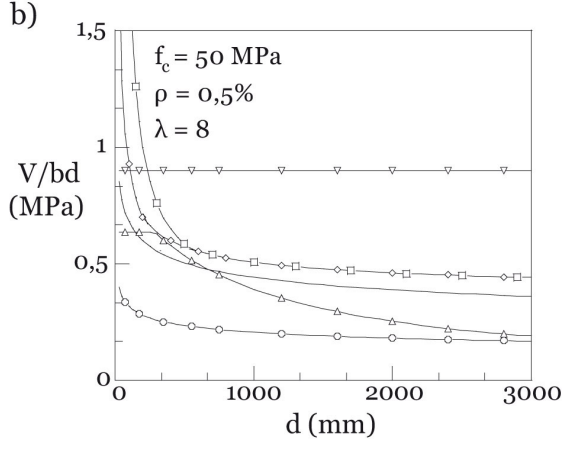

d)

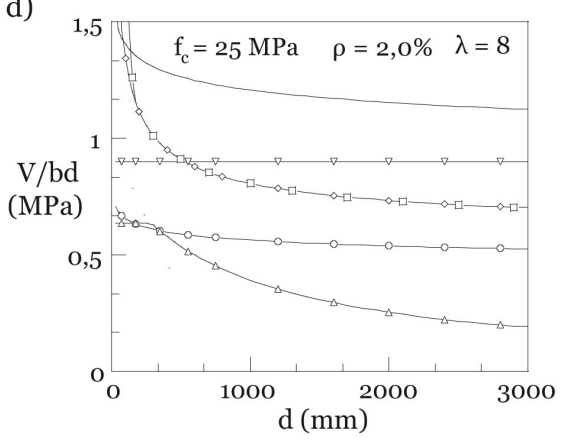

Figura: 7. Comparación de resultados de las ecuaciones [11] y [12] con las normativas EHE, EC2, Código Modelo y ACI: (a) fc $=25 \mathrm{MPa}, \rho=0,5 \%, \lambda=8$; (b) fc $=50 \mathrm{MPa}, \rho=0,5 \%$, $\lambda=8$; (c) fc $=25 \mathrm{MPa}, \rho=2,0 \%, \lambda=8$; (b) fc $=50 \mathrm{MPa}, \rho=2,0 \%, \lambda=8$.

dia es la que provoca que al hacer un análisis estadístico se reduzca tanto la carga de fallo para obtener un índice de fiabilidad según los requerimientos de la normativa. Este hecho justifica de por sí la necesidad de proponer modelos que nos permitan reproducir el fallo por tracción diagonal de forma correcta, con el fin de poder reducir la dispersión existente y de esta forma obtener ajustes que permitan una mayor seguridad en su aplicación.

\section{CONCLUSIONES}

El presente artículo presenta una metodología para obtener una formulación que tiene como fin evaluar la carga de fallo por tracción diagonal en vigas de hormigón armado sin cercos. La expresión surge de la aplicación de conceptos de la Mecánica de la Fractura para el estudio del efecto de tamaño combinados con observaciones experimentales. La carga de fallo viene dada por la suma de dos términos, uno que representa la resistencia del hormigón al progreso de la fisura, el cual está afectado por el efecto de tamaño, y un segundo término debido a la hiper-resistencia que se observa por la existencia de armado, el cual no está afectado por efecto de escala alguno. Para cuantificar el efecto de tamaño se han establecido dos modelos diferentes de comportamiento del hormigón, uno basado en Mecánica de Fractura Elástica Lineal y otro basado en el Ley de Efecto de Tamaño de Bažant. Los parámetros libres en las formulaciones se ajustaron me- diante los ensayos existentes de una base de datos de ensayos de vigas de hormigon armado sin cercos que se ha formado con diferentes fuentes bibliográficas.

Las expresiones obtenidas ajustan los resultados experimentales en todo el rango de tamaños de los ensayos de la base de datos que se ha tomado como referencia. Las comparaciones de los resultados obtenidos con otras formulaciones para evaluar la resistencia a cortante ponen de manifiesto la importancia de fundamentar las expresiones que se incluyen en normativas, ya que el rango de tamaños en ensayos realizados suele ser inferior al tamaño de los elementos de hormigón utilizados en construcción. Finalmente, se presenta una formulación de caracter práctico función de los parametros convencionalmente utilizados en el diseño de estructuras de hormigón. Esta formulación se compara con diversas normativas para comprobar la influencia tanto del efecto de la cuantía como de la resistencia del hormigón. La principal novedad de la expresión simplificada respecto a otras formulaciones es que la pendiente del efecto de tamaño varía en funcion de la cuantía de armado.

\section{AGRADECIMIENTOS}

Los autores agradecen la financiación recibida del Ministerio de Economía y Competitividad del Gobierno de España a través del proyecto MAT2012-35416. 


\section{REFERENCIAS}

(1) Zsutty, T.C. (1968). Beam shear strength prediction by analysis of existing data. ACI Structural Journal, 65(11): 943-951, doi: http://dx.doi.org/10.14359/7526.

(2) Rebeiz, K.S. (1999). Shear strength prediction for concrete members. Journal of Structural Engineering, 125(3): 301308. http://dx.doi.org/10.1061/(ASCE)0733-9445(1999)125:3(301).

(3) Okamura, H., Higai, T. (1980). Proposed design equation for shear strength of R.C. beams without web reinforcement. En Proceedings, Japan Society of Civil Engineering, 300:131-141.

(4) CEB (Comite Euro-International du Beton). (1991). CEB-FIP Model Code 199o, Final Draft. Lausanne.

(5) Ministerio de Fomento. (2008). EHE o8 Instrucción Española de Hormigón Estructural.

(6) Freire, M. J. (2004). Análisis de las formulaciones sobre la resistencia a cortante del hormigón. Informes de la Construccion, 497(57): 13-28, doi: http://dx.doi.org/10.3989/ic.2005.v57.i497.464.

(7) Vecchio, F. J., Collins, M. P. (1986). The modified compression field theory for reinforced concrete elements subjected to shear. ACI Journal, 83(2): 219-231.

(8) Vecchio, F. J., Collins, M. P. (1988). Predicting the response of reinforced concrete beams subjected to shear using the modified compression field theory. ACI Structural Journal, 85(3): 258-268.

(9) Bentz, E. C., Vecchio, F. J., Collins, M. P. (2006). Simplified modified compression field theory for calculating shear strength of reinforced concrete elements. ACI Structural Journal, 103(4): 614-624.

(10) Collins, M. P., Bentz, E. C., Sherwood, E. G. (2007). An adequate theory for the shear strength of reinforced concrete structures. En Morley Symposium on Concrete Plasticity and its Application (pp. 75-94). University of Cambridge.

(11) CSA Committee. (2004). A23.3-04 Design of Concrete Structures. Mississauga, ON, Canada: Canadian Standards Association.

(12) Muttoni, A., Ruiz, M. F. (2008). Shear strength of members without transverse reinforcement as function of critical shear crack width. ACI Structural Journal, 105(2): 163-172.

(13) AASHTO. (2008). AASHTO LRFD Bridge Design Specifications. American Association of State Highway and Transportation Officials.

(14) Fédération Internationale du Béton (FIB). (2012). Model Code 2010 - Final draft, Bulletins 65-66, Vols. 1-2. Lausanne, Switzerland: Fédération Internationale du Béton.

(15) Zararis, P. D., Papadakis, G. Ch. (2001). Diagonal shear failure and size effect in RC beams without web reinforcement. Journal of Structural Engineering, ASCE, 127(7): 733-742. http://dx.doi.org/10.1061/(ASCE)07339445(2001)127:7(733).

(16) Tureyen, A. K., Frosch, R. J. (2003). Concrete Shear Strength: Another Perspective. ACI Structural Journal, 100(5): 609-615, doi: http://dx.doi.org/10.14359/12802.

(17) Park, H-G, Choi, K-K, Wight, J. K. (2006). Strain-based shear strength model for slender beams without web reinforcement. ACI Structural Journal, 103(6): 783-793, doi: http://dx.doi.org/10.14359/18228.

(18) Park, H-G., Kang, S., Choi, K. K. (2013). Analytical model for shear strength of ordinary and prestressed concrete beams. Engineering Structures, 46: 94-103, doi: http://dx.doi.org/10.1016/j.engstruct.2012.07.015.

(19) Choi, K-K, Park, H-G, and Wight, J. K. (2007). Unified shear strength model for reinforced concrete beams-Part I: Development. ACI Structural Journal, 104(2): 142-152, doi: http://dx.doi.org/10.14359/18526.

(20) Choi, K-K, Park, H-G (2008). Unified shear strength model for reinforced concrete beams-Part II: Verification and simplified method. ACI Structural Journal, 104(2): 153-161, doi: http://dx.doi.org/10.14359/18527.

(21) Mari, A., Bairán, J. M., Cladera, A., Oller, E., Ribas, C. (2014). Shear-flexural strength mechanical model for the design and assessment of reinforced concrete beams. Structure and Infrastructure Engineering: Maintenance, Management, Life-Cycle Design and Performance, 11(11): 1399-1419, doi: http://dx.doi.org/10.1080/15732479.2014.964735.

(22) Bairán, J.M., Mari, A., Mohr, S. (2010). Estudio del comportamiento del hormigón armado ante esfuerzos normales y tangentes mediante modelos seccionales de interacción completa. Informes de la Construcción, 62(518): 67-77, doi: http://dx.doi.org/10.3989/ic.09.021.

(23) Perez-Caldentey, A., Padilla, P., Muttoni, A., Fernandez-Ruiz, M. (2011). Effect of load distribution and variable depth on shear resistance of slender beams without stirrups. ACI Structural Journal, 109(5):595-603.

(24) Reinhardt, H.W. (1981). Similitude of brittle fracture of structural concrete. En Advanced Mechanics of Reinforced Concrete, Delf, IASBE Colloquium (pp. 117-130).

(25) Ruiz, G., Elices, M., Planas, J. (1998). Experimental study of fracture of lightly reinforced concrete beams. Materials and Structures, 31(10): 683-691, doi: http://dx.doi.org/10.1007/BFo2480445.

(26) Ruiz, G., Carmona, J.R. (2006). Experimental study on the influence of the shape of the cross-section and of the rebar arrangement on the fracture of lightly reinforced beams. Materials and Structures, 39(3): 343-352, doi: http://dx.doi. org/10.1007/s11527-005-9006-7.

(27) Carmona. J.R. (2006). Study of Cracking Processes in Reinforced Concrete Elements (Tesis doctoral). Universidad de Castilla-La Mancha. https://ruidera.uclm.es/xmlui/handle/10578/2268.

(28) Carpinteri, A., Carmona, J.R., Ventura, G. (2007) Propagation of flexural and shear cracks through reinforced concrete beams by the bridged crack model. Magazine of Concrete Research, 59(10): 743-756, doi: http://dx.doi.org/10.168o/ macr.2007.59.10.743.

(29) Kani, G. N. J. (1967). How safe are our large reinforced concrete beams? ACI Journal Proceedings, 64: 123-141.

(30) Bažant, Z. P., Sun, H. H. (1987). Size effect in diagonal shear failure: Influence of aggregate size and stirrups. ACI Materials Journal, 84(4): 259-272. 
(31) Bažant, Z.P., Yu, Q. (2005) Designing against size effect on shear strength of reinforced concrete beams without stirrups: I. Formulation. Journal of Structural Engineering-ASCE, 131(12): 1877-1885, doi: http://dx.doi.org/10.1061/ (ASCE)0733-9445(2005)131:12(1877).

(32) Bažant, Z.P., Yu, Q. (2005). Designing against size effect on shear strength of reinforced concrete beams without stirrups: II. Verification and calibration. Journal of Structural Engineering-ASCE, 131(12): 1886-1897, doi: http://dx.doi. org/10.1061/(ASCE)0733-9445(2005)131:12(1886).

(33) Kim, J.K., Park, Y.D. (1996). Prediction of shear strength of reinforced concrete beams without web reinforcement. ACI Materials Journal 93(3): 213-222.

(34) Bažant, Z.P., Kazemi M. P. (1991). Size effect in diagonal shear failure. ACI Structural Journal, 88(3): $268-276$.

(35) Adebar, P., Collins M.P. (1996). Shear strength of members without transverse reinforcement. Canadian Journal of Civil Engineering, 23(1): 30-41, doi: http://dx.doi.org/10.1139/l96-004.

(36) Ahmad, S.H., Khaloo, A.R., Poveda, A. (1986). Shear capacity of reinforced high-strength concrete beams. ACI Journal, 83(2): 297-305, doi: http://dx.doi.org/10.14359/10433.

(37) Angelakos, D., Bentz, E.C., Collins, M.P. (2001). Effect of concrete strength and minimum stirrups on shear strength of large members. ACI Structural Journal, 98(3): 291-300, doi: http://dx.doi.org/10.14359/10220.

(38) Reineck, K.H., Kuchma, D.A., Kang Su Kin, Marx, S. (2003). Shear data base for reinforced concrete members without shear reinforcement. ACI Structural Journal 100(2): 240-249, doi: http://dx.doi.org/10.14359/12488.

(39) Bresler, B., Scordelis, A.C. (1963). Shear strength of reinforced concrete beam. Proceedings of ACI Journal, 60(1): 51-74, doi: http://dx.doi.org/10.14359/7842.

(40) Chana, P.S. (1981). Some Aspects of Modeling the Behavior of Reinforced Concrete under Shear Loading. Technical Report Nr. 543. Wexham Springs: Cement and Concrete Assoc.

(41) Collins, M.P., Kuchma, D. (1999). How safe are our large, lightly reinforced concrete beams, slabs and footings?, ACI Structural Journal, 96(4): 482-490.

(42) Diaz de Cossio, R., Siess, C.P. (1960). Behavior and strength in shear of beams and frames without web reinforcement. Proceedings of American Concrete Institute Journal, 56(2): 695-735, doi: http://dx.doi.org/10.14359/8118.

(43) Elzanaty, A. H., Nilson, A. H., Slate, F.O. (1986). Shear capacity of reinforced concrete beams using high-strength concrete. ACI Journal, Proceedings, 83(2): 290-296, doi: http://dx.doi.org/10.14359/10432.

(44) Grimm, R. (1997). Einfluß bruchmechanischer kenngrößen auf das biege- und schubtragverhalten hochfester betone. Darmstadt: Diss., Fachb. Konstr. Ingenieurbau der TH Darmstadt, 1996 und Berlin: DafStb H.477, Beuth Verlag GmbH.

(45) Islam, M.S., Pam, H.J., Kwan, A.K.H. (1998). Shear capacity of high-strength concrete beams with their point of inflection within the shear span. Proc of Inst of Civil Engr - Struct \& Bldgs, 128(1): 91-99, doi: http://dx.doi.org/10.168o/ istbu.1998.30038.

(46) Krefeld, W.J., Thurston, C.W. (1966). Studies of the shear and diagonal tension strength of simply supported reinforced concrete beams. Proceedings of ACI Journal, 63(4): 451-476, doi: http://dx.doi.org/10.14359/7633.

(47) Kuchma, D. (1999-2002). Shear Databank. Urbana-Champaign, IL: University of Illinois. http://cee.ce.uiuc.edu/kuchma/sheardatabank.

(48) Leonhardt, F., Walter, R. (1962). Schubversuche an einfeldriegen Stahlbeton-balken mit und ohne Schubbewehrung zur Ermittlung der Schubtragfähigkeit und der Oberen Schubspannungsgrenze, vol. 151.

(49) Moody, K.G., Viest, I.M., Elstner, R.C. (1954). Shear strength of reinforced concrete beams. Part 1 - Test of simple beams. Journal of the American Institute, 51(12): 317-332, doi: http://dx.doi.org/10.14359/11680.

(50) Morrow, J., Viest, I.M. (1957). Shear strength of reinforced concrete frame members without web reinforcement. Proceedings of ACI Journal, 53(3): 833-869, doi: http://dx.doi.org/10.14359/11558.

(51) Mphonde, A.G., Franz, G.C. (1984). Shear tests of high and low-strength concrete beams without stirrups. ACI Structural Journal, 81(4): 350-357, doi: http://dx.doi.org/10.14359/10690.

(52) Thorenfeldt, E. (1990). Shear capacity of reinforced high-strength concrete beams. HS Conc-Proc., 121: 129-154, doi: http://dx.doi.org/10.14359/2818.

(53) Bentz, E.C., Buckley, S. (2005). Repeating a classic set of experiments on size effect in shear of members without strirrups. ACI Structural Journal, 102(6): 832-838.

(54) Cladera, A., Mari, A.R. (2004). Shear design procedure for reinforced normal and high-strength concrete beams using artificial neural networks. Part I: beams without stirrups. Engineering Structures, 26(7): 917-926, doi: http://dx.doi. org/10.1016/j.engstruct.2004.02.010.

(55) Carmona, J.R., Ruiz, G., Del Viso, J.R. (2007). Mixed-mode crack propagation through reinforced concrete. Engineering Fracture Mechanics, 74(17): 2788-2809, doi: http://dx.doi.org/10.1016/j.engfracmech.2007.01.004.

(56) Bažant, Z.P. (1984). Size effect in blunt fracture: Concrete, rock, metal. Journal of Engineering Mechanics-ASCE, 110(4): 518-535, doi: http://dx.doi.org/10.1061/(ASCE)0733-9399(1984)110:4(518).

(57) Bažant, Z.P., Planas, J. (1998). Fracture Size Effect in Concrete and Other Quasibrittle Materials. Boca Raton: CRC Press.

(58) P.E. Petersson. (1981). Crack Growth and Development of Fracture Zone in Plain Concrete and Similar Materials. Report No. TVBM-1006, Lund, Sweden: Division of Building Materials, Lund Institute of Technology.

(59) Ruiz, G. (2001). Propagation of a cohesive crack crossing a reinforcement layer. International Journal of Fracture, 111(3): 265-282, doi: http://dx.doi.org/10.1023/A:1012260410704.

(60) Carpinteri, A., Carmona, J.R., Ventura G. (2011). Failure mode transitions in reinforced concrete beams - Part 2: Experimental tests. ACI Structural Journal, 108:286-293.

(61) Carpinteri, A., Carmona, J.R., Ventura G. (2011). Failure mode transitions in reinforced concrete beams - Part 1: Theoretical model. ACI Structural Journal, 108:277-285. 
(62) Carmona, J.R., Ruiz, G. (2014). Bond and size effects on the shear capacity of RC beams without stirrups. Engineering Structures, 66:45-56, doi: http://dx.doi.org/10.1016/j.engstruct.2014.01.054.

(63) ACI-318 (2011). Building Code Requirements for Structural Concrete (ACI 318-o8) and Commentary. Farmington Hills, MI, USA: ACI.

(64) Fernández-Montes, D., Díaz-Heredia, E., González-Valle, E. (2014). Evaluación del ajuste e introducción de la seguridad en el modelo experimental del EC-2 para estimar la capacidad a cortante en elementos lineales de hormigón armado sin armadura transversal. Hormigón y Acero, 65 (271): 71-84, doi: http://dx.doi.org/10.1016/So439-5689(14)500o8-3.

(65) CEN. (2004). Eurocode 2. Design of Concrete Structures, Part 1-1: General Rules and Rules for Buildings (EN1992-1-1). European Committee for Standardization (CEN). 